\title{
Error Tolerant MPC versus PI Control - a Crystallization Case Study
}

\author{
Luis Alberto Paz Suárez, Petia Georgieva, Member IEEE, Sebastião Feyo de Azevedo
}

\begin{abstract}
The present work is focused on a comparative study between the classical PI(D) control and an error tolerant modification of the nonlinear MPC approach implemented to a fed-batch sugar crystallization process. Two scenarios are considered: i) (nominal case) MPC versus PI assuming no disturbances or noise and ii) MPC versus PI with disturbances and noise due to original industrial data implemented in the tests. The results demonstrate that the MPC outperforms the PI control with respect to the final batch performance measures (less energy consumption, improved crystal size parameters and higher productivity). The usual high MPC computational costs are significantly reduced by the proposed error tolerant modification of the optimization procedure
\end{abstract}

\section{INTRODUCTION}

$\mathrm{D}$ URING the last decade the model based predictive control (MPC) became an attractive control strategy implemented in a variety of process industries [1]. First the linear MPC gained popularity as an industrial alternative and later on nonlinear cases as polymerization reactors [2] and reactive distillation columns [3] were reported as successfully MPC controlled processes. Despite these facts, MPC scheme still remains an interesting theoretical and practical control challenge particularly for batch processes [4]. The batch or fed-batch mode of operation is a typical production scheme for a large group of pharmaceutical, biotechnological, food and chemical processes. It is related with the formulation of a control problem in terms of economic or performance objective at the end of the process [5]. For example, the crystallisation quality is evaluated by the particle size distribution (PSD) at the end of the process which is quantified by two parameters - the final average (in

Manuscript received November 2, 2008. Several institutions contributed for this study: 1) Foundation of Science and Technology of Portugal, which financed the scholarship of investigation of doctorate SFR/16175/2004; 2) Laboratory for Process, Environmental and Energy Engineering (LEPAE), Department of Chemical Engineering, University of Porto; 3) Sugar refinery RAR, Portugal; 4) Company 30 de Noviembre, Pinar del Río, Cuba; 5) Institute of Electrical Engineering and Telematics (IEETA), University of Aveiro, Portugal. Special acknowledgements of the authors for the personnel of these institutions, who collaborated in the course of the present work.

L.A.P.S. is with the Department of Chemical Engineering, Faculty of Engineering, University of Porto, R. Dr. Roberto Frias, 4200-465 Porto, Portugal (corresponding author, phone: +351-22 508 1693; fax: +35122508 1632; e-mail: lpaz@fe.up.pt).

P.G. is with the Department of Electronics Telecommunications and Informatics and with the Institute of Electrical Engineering and Telematics (IEETA), University of Aveiro, Portugal (e-mail: petia@ua.pt).

S.F.A. is with the Department of Chemical Engineering, Faculty of Engineering, University of Porto, Portugal (e-mail: sfeyo@fe.up.pt). mass) particle size (MA) and the final coefficient of particle variation $(\mathrm{CV})$. The main challenge of the batch production is the large batch to batch variation of the final PSD [6]. This lack of process repeatability is caused mainly by improper control policy and results in product recycling and loss increase. MPC, being one of the approaches that inherently can cope with process constraints, nonlinearities, and different objectives derived from economical or environmental considerations, has the potential to overcome the problem of the lack of repeatability and drive the process to its optimal state of profit maximization and cost minimization. These problems are the main motivation for the present work, which is divided into the following sections. In section II the sugar production stages are shortly presented. In section III the crystallization phenomenological macro model is introduced. In section IV the error tolerant nonlinear MPC strategy is formulated and finally in section $\mathrm{V}$ and VI two scenarios with MPC and proportional integral (PI) controllers are tested and conclusions are drawn.

\section{II.PROCESS DESCRIPTION}

Sugar crystallisation occurs through the mechanisms of nucleation, growth and agglomeration. There are two basic types of sugar production: from cane sugar or from beet. The process considered in this work is of the first type and a typical industrial unit can be divided into the following sequential phases [7].

Charging: During the first phase the pan is partially filled with a juice containing dissolved sucrose (termed liquor). The initial liquid charged in the pan corresponds approximately to $40 \%$ of the total vessel height. The charge is usually performed by complete opening of the feeding valve until the level sensor indicates $40 \%$. Therefore, no special control policy is required at this stage.

Concentration: The next phase is the concentration. The liquor is concentrated by evaporation, under vacuum, until the supersaturation reaches a predefined value. At this value seed crystals are introduced into the pan to start the production of crystals. This is the beginning of the crystallisation phase.

Crystallization (main phase): At this phase as evaporation takes place further liquor is added to the pan in order to guarantee crystal growth at a controlled supersaturation level and to increase the sugar content of the pan. Near to the end of this phase and for economical reasons, the liquor is 
replaced by other juice of lower purity (termed syrup).

Tightening: The fourth phase consists of tightening which is principally controlled by evaporation capacity. The pan is filled with a suspension of sugar crystals in heavy syrup, which is dropped into a storage mixer. At the end of the batch, the massecuite undergoes centrifugation, where final refined sugar is separated from (mother) liquor that is recycled to the process.

Sugar production is still a very heuristically operated process, with classical proportional integral and eventually derivative (PID) controllers being the most typical solution. However, the industrial partners ((Sugar Refinery RAR, Portugal, Company 30 de Noviembre, Pinar del Río, Cuba) agreed that an optimized operation policy might result in reduction of the recycled batches and thus in reduction of energy and material loss. These problems constitute the main motivation of the present work with the objective to derive an efficient optimized operation strategy for the process in hand. The second motivation is related with the fact that the crystallization phenomenon is typical for a great number of industrial processes such as for example in the pharmaceutical and food engineering. Therefore, the strategy formulated for this case study can be further extended and applied for other crystallization based processes.

Since the different phases of the sugar production are comparatively independent and moved by distinct driving forces, it is not worth to look for a single controller for all of them. Therefore, individual MPC controllers for each stage where it seems appropriate, was the adopted framework (see Table I).

\section{CRYSTALliZATION MACRO MODEL}

The general phenomenological model of the fed-batch crystallization process consists of mass, energy and population balances [8]. While the mass and energy balances are common expressions in many chemical process models, the population balance is related with the crystallization phenomenon which is still an open modeling problem.

\section{A. Mass balance}

The mass of all participating solid and dissolved substances are included in a set of conservation mass balance equations

$\dot{M}=f(M(t), F(t), P 1), \quad 0 \leq t \leq t_{f}, \quad M(0)=M_{0}$

where $M(t) \in R^{q}$ and $F(t) \in R^{m}$ are the mass and the flow rate vectors, with $q$ and $m$ dimensions respectively, and $t_{f}$ is the final batch time. $P 1$ is the vector of physical parameters as density, viscosity, purity, ect.

\section{B. Energy balance}

The general energy (E) balance model is

$$
\dot{E}=f(E(t), M(t), F(t), P 2), \quad 0 \leq t \leq t_{f}, \quad E(0)=E_{0}
$$

where $P 2$ incorporates the enthalpy terms and specific heat capacities derived as functions of physical and thermodynamic properties.

\section{Population balance}

Mathematical representation of the crystallization rate can be achieved through basic mass transfer considerations or by writing a population balance represented by its moment equations. Employing a population balance is generally preferred since it allows to take into account initial experimental distributions and, most significantly, to consider complex mechanisms such as those of size dispersion and/or particle agglomeration/aggregation. Hence

$$
\begin{aligned}
& \dot{\eta}_{i}=f\left(\eta_{i}(t), \tilde{B}_{0}, G, \beta^{\prime}\right), 0 \leq t \leq t_{f}, i=0,1,2, \ldots \\
& \eta_{i}(0)=\eta_{i 0}
\end{aligned}
$$

where $\eta_{i}$ is the $i$-th moment of the mass-size particle distribution function, $\tilde{B}_{0}, G$ and $\beta$, are the kinetic variables nucleation rate, linear growth rate and the agglomeration kernel, respectively. The PSD measures: the final average (in mass) particle size (MA) and the final coefficient of particle variation (CV) are derived from (3) as follows

$$
\begin{aligned}
& \mathrm{MA}=\eta_{1} / \eta_{0} \\
& \mathrm{CV}=\left(\eta_{0} \eta_{2} / \eta_{1}^{2}-1\right)^{1 / 2}
\end{aligned}
$$

For more details with respect to the detailed process model see [8].

\section{GENERAL VERSUS ERROR TOLERANT MPC}

\section{A. General MPC problem formulation}

Nonlinear model predictive control (NMPC) is an optimisation-based multivariable constrained control technique that uses a nonlinear dynamic model for the prediction of the process outputs [9]. At each sampling time the model is updated on the basis of new measurements and state variables estimates. Then the open-loop optimal manipulated variable moves are computed over a finite (predefined) prediction horizon with respect to some performance index, and the manipulated variables for the subsequent prediction horizon are implemented. Then the prediction horizon is shifted or shrunk by usually one sampling time into the future, and the previous steps are 
repeated.

TABLE I

SUMMARY OF THE OPERATION STRATEGY FOR THE SUGAR CRYSTALLIZATION PROCESS

\begin{tabular}{|c|c|c|}
\hline Stage & Action & Control \\
\hline Charge & $\begin{array}{l}\text { The steam valve is closed. } \\
\text { The stirrer is off. } \\
\text { The vacuum pressure changes from } 1 \text { to } 0.23 \text { bar. } \\
\text { The vacuum pressure reaches } 0.5 \text { bar, feeding starts with max rate. } \\
\text { Liquor covers } 40 \% \text { of the vessel height }\end{array}$ & $\begin{array}{l}\text { No control } \\
\text { The feed valve is completely } \\
\text { open }\end{array}$ \\
\hline Concentration & $\begin{array}{l}\text { The vacuum pressure stabilizes around } 0.23 \text { bar. } \\
\text { The stirrer is on. } \\
\text { The volume is kept constant . } \\
\text { The steam flowrate increases to } 2 \mathrm{~kg} / \mathrm{s} \\
\text { The supersaturation reaches } 1.06 \text {, the feeding is closed, the steam flowrate is } \\
\text { reduced to } 1.4 \mathrm{~kg} / \mathrm{s}\end{array}$ & $\begin{array}{l}\text { Control loop } 1 \\
\text { Controlled variable: Volume; } \\
\text { Manipulated variable: liquor } \\
\text { feed flowrate }\end{array}$ \\
\hline $\begin{array}{l}\text { Seeding and } \\
\text { setting the } \\
\text { grain }\end{array}$ & $\begin{array}{l}\text { The supersaturation reaches } 1.11 . \\
\text { Seed crystals are introduced. } \\
\text { The steam flowrate is kept at the minimum for two minutes. }\end{array}$ & $\begin{array}{l}\text { No control } \\
\text { The feed valve is closed }\end{array}$ \\
\hline $\begin{array}{l}\text { Crystallization } \\
\text { with liquor } \\
\text { (phase 1) }\end{array}$ & $\begin{array}{l}\text { The steam flowrate is kept around } 1.4 \mathrm{~kg} / \mathrm{s} \text {. } \\
\text { The supersaturation is controlled at the set point } 1.15 \text {. }\end{array}$ & $\begin{array}{l}\text { Control loop } 2 \text { Controlled } \\
\text { variable: supersaturation } \\
\text { Manipulated variable: liquor } \\
\text { feed flowrate }\end{array}$ \\
\hline $\begin{array}{l}\text { Crystallization } \\
\text { with liquor } \\
\text { (phase 2) }\end{array}$ & $\begin{array}{l}\text { The volume of crystallizer reaches } \approx 22 \mathrm{~m}^{3} \\
\text { The feed valve is closed. } \\
\text { The supersaturation is controlled at the set point } 1.15 \text {. } \\
\text { The stirrer power reaches } 20.5 \mathrm{~A} \text {. }\end{array}$ & $\begin{array}{l}\text { Control loop } 3 \text { Controlled } \\
\text { variable: supersaturation } \\
\text { Manipulated variable: steam } \\
\text { flowrate }\end{array}$ \\
\hline $\begin{array}{l}\text { Crystallization } \\
\text { with syrup }\end{array}$ & $\begin{array}{l}\text { The steam flowrate is kept around the maximum of } 2.75 \mathrm{~kg} / \mathrm{s} \text {. (hard constraint). } \\
\text { The volume fraction of crystals is kept at the set point } 0.45 \text {. } \\
\text { The volume reaches its maximum value }\left(30 \mathrm{~m}^{3}\right) \\
\text { The feed valve is close. }\end{array}$ & $\begin{array}{l}\text { Control loop } 4 \text { Controlled } \\
\text { variable: volume fraction of } \\
\text { crystals. Manipulated variable: } \\
\text { syrup feed flowrate }\end{array}$ \\
\hline Tightening & $\begin{array}{l}\text { The stirrer power reaches the maximum value of } 50 \text { A (hard constraint). } \\
\text { The steam valve is closed. } \\
\text { The stirrer and the barometric condenser are stopped. }\end{array}$ & No control \\
\hline
\end{tabular}

The optimal control problem in the NMPC framework can be mathematically formulated as:

$\min _{u_{\min } \leq u(t) \leq u_{\max }} J=\varphi(x(t), u(t), P)$

subject to:

$\dot{x}=f(x(t), u(t), P), \quad 0 \leq t \leq t_{f}, \quad x(0)=x_{0}$

$y(t)=h(x(t), P)$

$g_{j}(x)=0, \quad j=1,2, \ldots \ldots . p$

$v_{j}(x) \leq 0, \quad j=1,2, \ldots . . l$

where (5) is the performance index, (6) is the process model, function $f$ is the state-space description, function $h$ is the relationship between the output and the state, $P$ is the vector of possibly uncertain parameters and $t_{f}$ is the final time. $x(t) \in R^{n}, u(t) \in R^{m}$ and $y(t) \in R^{p}$ are the state, the manipulated input and the control output vectors, respectively. The manipulated inputs, the state and the control outputs are subject to the following constraints, $x(t) \in \mathrm{X}, u(t) \in \mathrm{Z}, y(t) \in \mathrm{Y}$ in which $\mathrm{X}, \mathrm{Z}$ and $\mathrm{Y}$ are convex and closed subsets of $R^{n}, R^{m}$ and $R^{p} . g_{j}$ and $v_{j}$ are the equality and inequality constraints with $p$ and $l$ dimensions respectively.

\section{B. Error tolerant digital nonlinear MPC control framework (main contribution)}

Discrete process model. Considering the discrete nature of the on-line control problem the continuous time optimization problem involved in the NMPC formulation is solved by formulating a discrete approximation to it, that can be handled by conventional nonlinear programming (NLP) solvers. The time horizon $t=\left\lfloor t_{0}, t_{f}\right\rfloor$ is divided into equally spaced time intervals $\Delta t$, with discrete time steps $t_{k}=t_{0}+k \Delta t$ and $k=0,1, \ldots, N$. The process model (6) is discretised as follows:

$$
\begin{aligned}
& x(k+1)=F[(x(k), u(k), P] \\
& y_{p}(k)=h[(x(k), P]
\end{aligned}
$$

Process input constraints arise due to actuator limitations such as saturation and rate-of-change restrictions and can be expressed as

$$
\begin{aligned}
& u_{\text {min }} \leq u(t+k) \leq u_{\max }, \quad k=1,2, \ldots H_{c} \\
& \Delta u_{\text {min }} \leq \Delta u \leq \Delta u_{\max }
\end{aligned}
$$


where $u_{\min }$ and $u_{\max }$ are the minimum and the maximum values of the input, $\Delta u_{\min }$ and $\Delta u_{\max }$ are the minimum and the maximum values of the rate-of-change of the same input.

Process output constraints are usually associated with operational limitations such as equipment specifications and safety considerations and can be expressed as:

$y_{\text {min }} \leq y_{p}(t+k) \leq y_{\text {max }}, \quad k=1,2, \ldots H_{p}$

We propose a modification of the general MPC formulation (5), where the optimization is performed based on the following performance index

$$
u(t+k)= \begin{cases}u:\left\{\begin{array}{ll}
\min _{\left[u(t+k), u\left(t+k+1, \ldots \ldots u\left(t+H_{c}\right)\right)\right]} J= \\
\lambda_{1} \sum_{k=1}^{H_{p}}(e(t+k))^{2}-\lambda_{2} \sum_{k=1}^{H_{c}}(\Delta u)^{2}
\end{array}, \text { if }|e(t)|>\alpha\right. \\
u^{*} & \text { if }|e(t)|<\alpha\end{cases}
$$

where $e(t)=r(t)-y(t)$,

$$
\Delta u=u(t+k-1)-u(t+k-2)
$$

subject to constraints (9) and (10). $y(t) \in R^{p}$ are the process measurements.

Equation (11) is a particular discrete format of the general performance index defined by (5) [20]. We denote it as an error tolerant $M P C$ formulation because the optimization is performed only when the error $e(t+k)$ is bigger than a predefined $\alpha$ value. In order to reduce the computational burden when the absolute error $e(t+k)$ is smaller than $\alpha$ the control action is equal to $u^{*}$ which is the last value computed before the error enters the $\alpha$ strip. $\alpha$ is a design parameter and its choice is crucial for achieving a good compromise between bearable computational costs and acceptable tracking error. The prediction horizon $H_{p}$ is the number of time steps over which the prediction errors are minimized and the control horizon $H_{c}$ is the number of time steps over which the control increments are minimized, $r$ is the desired response and $y_{p}$ is the prediction model response.

$u(t+k), u(t+k+1), \ldots . . u\left(t+H_{c}\right)$ are tentative values of the future control signal, which are limited by $u_{\min }$ and $u_{\max }$. Parameters $\lambda_{1}$ and $\lambda_{2}$ determine the contribution of the output error and the contribution of the control increments respectively on the performance index. In all control tests summarized in section $\mathrm{V} \lambda_{1}$ was set to 1 , as a normalized value for the importance of the first term. For the choice of $\lambda_{2}$ an empirical formula was deduced as follows,

$$
\left(u_{\max }-u_{\min }\right)^{2} \cdot \lambda_{2}=e_{p \max } \cdot P / 100
$$

where $\mathrm{P}$ defines the desired contribution of the second term in $(11)(0 \% \leq \mathrm{P} \leq 100 \%)$ and

$$
e_{p \max }=\max \left(\left(r-y_{\max }\right)^{2},\left(r-y_{\text {min }}\right)^{2}\right)
$$

The intuition behind (12-13) is to make the two terms of (11) compatible when they are not previously normalized and to overcome the problem of different numerical ranges of the two terms.

Note that, the main reason for the $\alpha$ error modification of the classical MPC is the expectation to reduce the computational burden. MPC controller requires a significant amount of on-line computation, since the optimization (5) is performed at each sample time to compute the optimal control input. However, the $\alpha$ error tolerance proposed by the modification (8) reduces significantly the computational efforts.

\section{V.CONTROL TESTS}

\section{A. PID controllers}

First the PID parameters were tuned, where $k_{p}, \tau_{i}, \tau_{d}$ are related with the general PID terminology as follows:

$u(t+k)=k_{p}\left[e(t+k)+\frac{\Delta_{s}}{\tau_{i}} \cdot \sum_{i=0}^{k} e(t+i)+\frac{\tau_{d}}{\Delta_{s}} \cdot(e(t+k)-e(t+k-1))\right]$

Since the process is nonlinear, classical (linear) tuning procedures were substituted by a numerical optimization of the integral (or sum in the discrete version) of the absolute error (IAE):

$I A E=\sum_{k=1}^{N}\left|r(t+k)-y_{p}(t+k)\right|$

Equation (15) was minimized in a closed loop framework between the discrete process model and the PID controller. For each parameter an interval of possible values was defined based on empirical knowledge and the process operator expertise. A number of gradient (Newton-like) optimization methods were employed to compute the final values of each controllers summarized in Table II. All methods concluded that the derivative part of the controller is not necessary. Thus, a PI controller was analyzed in the next tests. 
TABLE II

OPTIMIZED PID PARAMETERS (EQ. 14) FOR THE CONTROL LOOPS DEFINED IN TABLE I

\begin{tabular}{lllll}
\hline \hline & $\begin{array}{l}\text { Control } \\
\text { Loop 1 }\end{array}$ & $\begin{array}{l}\text { Control } \\
\text { Loop 2 }\end{array}$ & $\begin{array}{l}\text { Control } \\
\text { Loop 3 }\end{array}$ & $\begin{array}{l}\text { Control } \\
\text { Loop 4 }\end{array}$ \\
\hline$k_{p}$ & 0.05 & -0.5 & 20 & -0.01 \\
$\tau_{i}$ & 30 & 40 & 10 & 70 \\
$\tau_{d}$ & 0 & 0 & 0 & 0 \\
\hline \hline
\end{tabular}

\section{B. MPC parameters $\left(\alpha, H_{p}, H_{c}\right)$}

The values of $\alpha$ in (11) were defined based on empirical process knowledge and are summarized in Table III.

TABLE III

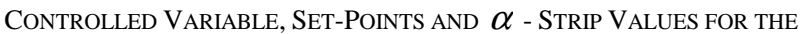
CONTROL LOOPS DEFINED IN TABLE I

\begin{tabular}{lcccc}
\hline \hline $\begin{array}{l}\text { Control Loop } \\
(\text { CL) /controlled } \\
\text { variable }\end{array}$ & $\begin{array}{c}\text { CL 1 / } \\
\text { Volume }\end{array}$ & $\begin{array}{c}\text { CL 2/ } \\
\text { Supersatur. }\end{array}$ & $\begin{array}{c}\text { CL 3/ } \\
\text { Supersatur. }\end{array}$ & $\begin{array}{c}\text { CL 4/ } \\
\text { Fraction } \\
\text { of crystals }\end{array}$ \\
\hline Set-point & 12.15 & 1.15 & 1.15 & 0.43 \\
$\alpha$-strip & 0.02 & 0.01 & 0.001 & 0.001 \\
\hline \hline
\end{tabular}

The operation strategy, summarized in Table I and implemented by a sequence of MPC (Table III) or PI controllers (Table II) is comparatively tested in Matlab environment [10]. A process simulator was developed based on a detailed phenomenological model [8]. The output predictions are computed by the discretized model (8). The choice of the prediction horizon $H_{p}$ is a function of the process time constant. Since the full process response is almost complete within $50 \mathrm{~s}$. $\left(H_{p} * \Delta t_{s}=50\right)$ and the sampling interval is $\Delta t_{s}=10 \mathrm{~s}$, the prediction horizon was set at $H_{p}=5$ and the control horizon as $H_{c}=2$.

\section{Results}

Scenario 1 (nominal case): MPC versus PI assuming no disturbances or noise. The main operation parameters are kept constant (see Table IV).

Scenario 2: MPC versus PI in the presence of realistic disturbances and noise. Instead of analytical expressions for the vacuum pressure, brix and temperature of the feed flow, pressure and temperature of the steam, original industrial data without any preprocessing is embedded into the model.

Time trajectories of the controlled and control (manipulated) variables for the main crystallization (2 and 3) control loops are depicted in Fig. 1. Note that MPC is more efficient for loop 2 while PI outperforms MPC in loop 3.
However, in average both control strategies (PI and MPC) are equally successful in keeping the controlled outputs around their set points. The set-points, for example supersaturation $=1.15$, are chosen empirically as the values referenced by the particular sugar refinery process considered and eventually they might not be the optimal values. Due to space limits the results for the other control loops are not shown in this paper but they look similarly in the sense that over the sequence of control loops MPC do not bring significant tracking improvements to justify its use. However, the main benefits of the MPC strategy are with respect to the batch end point performance. The results of the two scenarios are summarized in Table IV. Note that with respect to AM, the MPC has slightly better results that could probably disappear in the real system due to the measurement error effect. More realistic advantage of MPC is the improvement of CV in Scenario 2 (Table IV) and the lower energy consumption in Scenario 1. These improvements are achieved with less energy quantified by the kilograms of vapor consumed. Moreover the MPC strategy led to higher quantity of sugar for approximately the same batch duration (scenario 2). There is a clear tendency of final batch process improvement when the proposed MPC control is applied.

Remark: Using the same amount of raw material in both cases will improve the comparison between the two control paradigms, however during a normal operation cycle these quantities vary. For example the liquor consumed is usually not fixed, its quantity depends on the time it takes to achieve the supersaturation set point.

A comparison between the classical and the modified MPC proposed in this work was also made with respect to the anticipated reduction of computational efforts. In Fig. 2 are depicted the CPU times per iteration along the process duration for both methods. The small error tolerance influences directly the computational time and for about half of the iterations the time is much less. The work reported here is at an initial stage and more study is required to get a comprehensive insight into the effect of the $\alpha$ parameter.

\section{CONCLUSIONS}

The main contribution of the work is a modified digital MPC control where the optimization is only performed when the tracking error is above a predefined level $\alpha$. This new algorithm is implemented for the challenging nonlinear process of sugar production and compared with traditionally applied PI controller. An operation strategy with four control loops was formulated, with individual MPC or PI controllers, and different simulation scenarios considered. The results has shown that the error tolerant MPC and the PI controllers perform equally good with respect to the individual loop tracking error but the MPC definitely outperforms the PI strategy with respect to the final batch performance measures 
(less energy consumption, improved crystal size parameters and higher productivity).

TABLE IV

PI \&MPC FINAL BATCH PERFORMANCE MEASURES

\begin{tabular}{lllll}
\hline \hline & \multicolumn{2}{l}{ Scenario 1 } & \multicolumn{2}{l}{ Scenario 2 } \\
\hline Performance measures & PI & MPC & PI & MPC \\
\hline AM (mm) (reference 0.58) & 0.59 & 0.58 & 0.6 & 0.59 \\
CV (\%) (reference 30) & 31.34 & 31.2 & 31.12 & 30 \\
Batch duration & $87: 55$ & $89: 00$ & $94: 26$ & $94: 30$ \\
Liquor consumption , ${ }^{3}$ & 38.54 & 38.84 & 39.18 & 39.6 \\
$\begin{array}{l}\text { Energy consumption } \\
\text { (Quantity of vapor in kg) }\end{array}$ & 9398 & 9261 & 9937 & 9849 \\
$\begin{array}{l}\text { Quantity of sugar } \\
\text { production ( t) }\end{array}$ & 21.707 & 22.03 & 21.72 & 22.09 \\
\hline \hline
\end{tabular}

Controlled Variable 2-3 (Supersaturation)

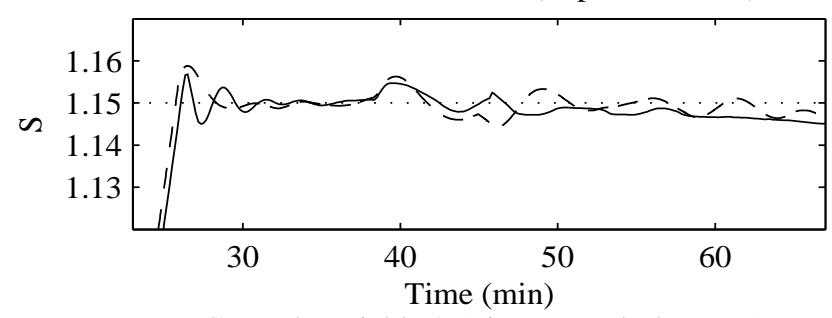

Control Variable 2 (Liqour Feed Flowrate)

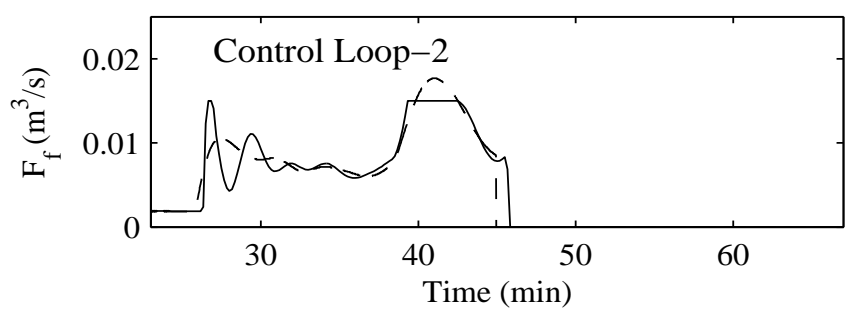

Control Variable 3 (Steam Flowrate)

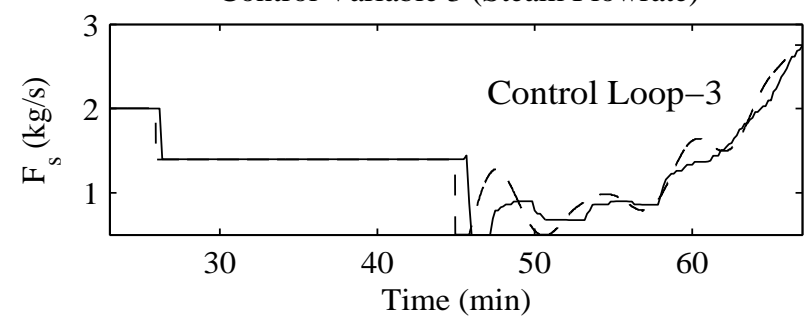

Fig.1. Time trajectories of the controlled ( $\mathrm{S}$-supersaturation) and control variables (Ff- feed flowrate, Fs -steam flow rate) for the 2 and 3 control loops. Dashed lines - PI control; Full lines - error tolerant MPC (a)

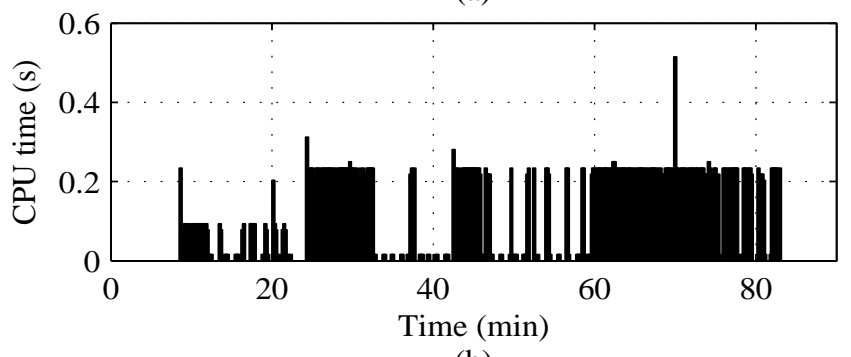

(b)

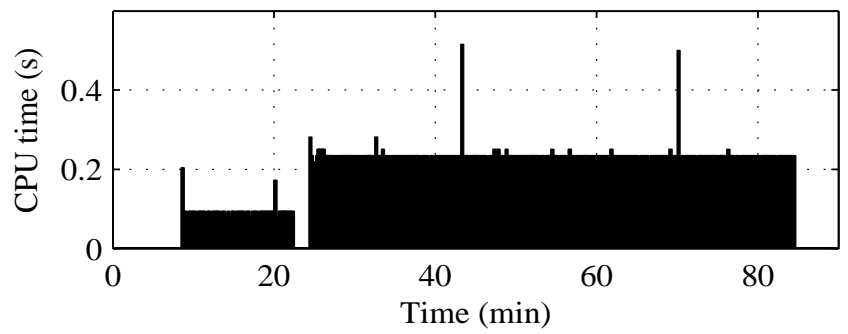

Fig. 2. CPU time per iteration along the process duration. (a) -Error tolerant MPC -Eq. (11); (b) - Classical MPC

\section{REFERENCES}

[1] E. F. Camacho and C. Bordodns (2004) Model Predictive Control in the process industry, London, Springer-Verlag.

[2] H. Seki, M. Ogawa, S. Ooyama, K. Akamatsu, M. Ohshima and W. Yang (2001) Industrial application of a nonlinear model predictive control to polymerization reactors, Control Engineering Practice, 9, 819-828

[3] L. S. Balasubramhanya and F. J. Doyle, (2000) Nonlinear modelbased control of a batch reactive distillation column. Journal of Process Control, 10, 209-218.

[4] F. Allgower, R. Findeisen and Z. K. Nagy (2004) Nonlinear model predicitve control: From theory to application. Journal of Chinese Institute of Chemical Engineers, 35, 299-315.

[5] Z. K. Nagy and R. D. Braatz (2003) Robust nonlinear model predictive control of batch processes. AIChE J, 49 (7), 1776-1786.

[6] A. Simoglou, P. Georgieva, E. Martin, E. B., J. Morris and S. F. de Azevedo (2005) On-line Monitoring of a Sugar Crystallization Process. Computers \& Chemical Engineering, 29, 1411-1422.

[7] V. Galvanauskas, P. Georgieva, and S. F. de Azevedo. (2006) Dynamic Optimisation of Industrial Sugar Crystallization Process based on a Hybrid (mechanistic+ANN) Model. IEEE World Congress on Computational Intelligence. 16-21 July 2006, Vancouver, Canada.

[8] P. Georgieva,, M. J . Meireles and S. F. de Azevedo (2003) Knowledge Based Hybrid Modeling of a Batch Crystallization When Accounting for Nucleation, Growth and Agglomeration Phenomena. Chemical Engineering Science, 58, 3699-3707.

[9] J. A. Rossiter (2003) Model based predictive control. A practical approach, New York, CRC Press.

[10] A. Bemporad, M. Morari and N. L. Ricker , (2005) User's Guide: Model Predictive Control Toolbox for use with MatLab, Version 2. The MathWorks Inc. 